EXTENDED REPORT

\title{
Ocular blood flow alteration in glaucoma is related to systemic vascular dysregulation
}

\author{
M Emre, S Orgül, K Gugleta, J Flammer
}

Br J Ophthalmol 2004;88:662-666. doi: 10.1136/bjo.2003.032110

See end of article for authors' affiliations ....................

Correspondence to: Selim Orgül, $M D$, University Eye Clinic Basel, Mittlere Strasse 91, PO Box, 4012 Basel, Switzerland; sorgul@magnet.ch

Accepted for publication 1 October 2003

\begin{abstract}
Aims: To investigate the source of ocular blood flow alterations in glaucoma.
Methods: In 56 patients with open angle glaucoma, blood flow parameters were obtained from both eyes in the ophthalmic and central retinal artery by means of colour Doppler imaging, as well as in the choroidal circulation and the neuroretinal rim of the optic nerve by means of laser Doppler flowmetry. Based on these haemodynamic parameters, a cluster analysis (two groups) was performed and differences with regard to risk factors were assessed between clusters.

Results: Ocular blood flow data in the two clusters indicated that the two groups (cluster $1=26$ patient with higher blood flow values; cluster $2=30$ patients with lower blood flow values) differed mainly in choroidal and optic nerve blood flow. No differences in sex distribution, propensity to have normal tension glaucoma, age, endothelin-1 plasma levels, visual field damage, intraocular pressure, or systemic blood pressure parameters were observed between the two clusters. However, 12 patients $(46 \%)$ from the cluster with high ocular blood flow values showed a vasospastic response in nailfold capillaroscopy, while such a response was observed in 24 patients $(80 \%)$ of the cluster with low ocular blood flow values. This difference in vasospastic propensity was statistically significant $(p=0.0121)$.

Conclusions: Ocular blood flow alterations in glaucoma patients seem, at least partly, to be related to a systemic vascular dysregulation.
\end{abstract}

O cular blood flow alterations in glaucoma have been described in many studies. Unfortunately, various and numerous techniques appraising different aspects of the ocular circulation were used, hampering a clear understanding of the role of blood flow in glaucoma. A number of conditions such as congenital glaucoma, angle closure glaucoma, or secondary glaucomas clearly show that increased intraocular pressure is sufficient to lead to glaucomatous optic neuropathy. ${ }^{1}$ Conversely, the existence of normal tension glaucoma on one hand and patients with ocular hypertension (increased IOP without recognisable damage) on the other, indicate that other factors might also be involved in the pathogenesis of glaucomatous optic neuropathy, either damaging the eye directly or rendering it more sensitive to IOP. Of special interest are vascular factors. ${ }^{2}$ However, the interpretation of the available data is difficult. Blood flow reduction may, at least partly, be due to secondary adaptation to a reduced demand. Furthermore, blood flow alterations have been described in various parts of the ocular circulation and it remains unclear how circulatory disorders in parts of the eye other than the anterior optic nerve may encroach on axonal survival. Finally, the influence of additional factors such as sex, ${ }^{3}$ plasma levels of endothelin$1,{ }^{45}$ systemic blood pressure, ${ }^{6-10}$ and vasospasm ${ }^{11}$ remains to be clarified. In this clinical study, a cluster analysis based on ocular haemodynamic parameters was performed in patients with open angle glaucoma, and differences with regard to risk factors were assessed between clusters.

\section{PATIENTS AND METHODS \\ Patients}

We evaluated 56 consecutive patients with primary open angle glaucoma in a cross sectional study during the period between 1997-2000. All procedures conformed to the Declaration of Helsinki. Patients with closed iridocorneal angles, evidence of secondary glaucoma, pseudoexfoliation, pigmentary dispersion, a history of intraocular surgery, any form of retinal or neuro-ophthalmological disease that could result in visual field defects, or with a history of chronic systemic disease, including diabetes mellitus, systemic hypertension, or occlusive vascular disorders, were excluded from this study. Smokers and patients taking local or systemic drugs likely to influence intraocular pressure or ocular blood flow were excluded. Patients with ocular media opacification precluding proper visualisation of fundus details or ametropia above plus or minus 3 dioptres spherical equivalent or 1 dioptre astigmatism were excluded. Selected patients did not receive any antiglaucoma medication. All patients had typical glaucomatous disc and visual field damage. After approval by the ethics committee, informed consent for the use of their clinical data in a scientific publication was obtained from the patients. The glaucoma patients underwent a diurnal IOP curve (Goldmann applanation tonometry at $6 \mathrm{am}$, before arising from bed, 8 am $11 \mathrm{am}$, $4 \mathrm{pm}$, and $10 \mathrm{pm}$ ). Plasma endothelin-1 levels were determined by specific radioimmunoassay as previously described. ${ }^{12}$ Visual field examinations were performed with the program $\mathrm{Gl}^{13}$ on the Octopus visual field analyser (Interzeag, Schlieren, Switzerland). Minimal criteria for glaucomatous visual field defects were a cluster of three points (except rim points) in at least one hemifield reduced by $5 \mathrm{~dB}$ or greater, and including at least one point reduced by $10 \mathrm{~dB}$ or greater; a cluster of two points reduced by $10 \mathrm{~dB}$ or greater; or three adjacent points on the nasal horizontal meridian that differed by $5 \mathrm{~dB}$ or greater from their mirror points on the opposite side of the meridian. Patients with poor visual field reliability (false positive or false negative errors exceeding 25\%) were not enrolled. Enrolled patients

Abbreviations: $\mathrm{CDI}$, colour Doppler imaging; $\mathrm{CRA}$, central retinal artery; DBP, diastolic blood pressure; EDV, end diastolic velocity; IOP, intraocular pressure; LDF, laser Doppler flowmetry; OA, ophthalmic artery; OPP, ocular perfusion pressure; PSV, peak systolic velocity; SBP, systolic blood pressure 
had $3 \mathrm{~mm}$ or larger pupil diameters when their fields were plotted.

\section{Systemic blood pressure}

All patients had a 24 hour blood pressure monitor (Mobil-OGraph, IEM GmbH, Stolberg, Germany). During daytime (from $8 \mathrm{am}$ to $10 \mathrm{pm}$ ) and night-time (from $10 \mathrm{pm}$ to $8 \mathrm{am}$ ) the measurement intervals were 30 minutes. Recordings were performed in the hospital, so that the patients were all under comparable conditions during the 24 hour blood pressure measurement period. The mean systolic (SBP), and diastolic blood pressure (DBP) for daytime and night-time were computed for each patient. The blood pressure dip, representing the fall in blood pressure during night-time expressed as a percentage of the average daytime reading level, was determined for SBP and DBP in each patient. Using average IOP of the diurnal tension curve and mean SBP and DBP during daytime, ocular perfusion pressure (OPP) was estimated for each eye $\left(\mathrm{OPP}=2 / 3 \times\left[\begin{array}{ll}2 / 3 & \mathrm{DBP} \\ 2 & 2 / 3 \mathrm{SBP}\end{array}\right]-\right.$ IOP).

\section{Nailfold capillaroscopy}

All the patients underwent microscopic examination of their nailfold capillaries, as described previously. ${ }^{11}$ Briefly, a light microscope is coupled to a television monitor that is in turn coupled to a video recorder, allowing the observed blood flow to be videotaped for later analysis. During capillaroscopy, the nailfold area is cooled to $-15^{\circ} \mathrm{C}$ for 60 seconds by rapidly decompressing carbon dioxide. The examination is performed in a room with a constant temperature of about $23^{\circ} \mathrm{C}$ (range $21^{\circ} \mathrm{C}-25^{\circ} \mathrm{C}$ ). During cooling, the blood in the capillaries sometimes stops flowing. Digital vasospasm is defined as a closing of one or more visible capillaries, with a mean stoppage time of longer than 12 seconds.

\section{Colour Doppler sonography}

All the glaucoma patients underwent blood flow velocity assessment of their ophthalmic artery (OA) and their central retinal artery (CRA) by means of colour Doppler imaging (CDI) as described previously. ${ }^{14}$ The same experienced sonographer performed all retrobulbar CDI examinations by means of a colour Doppler imaging device (Siemens Albis AG, Zürich, Switzerland) using a $7.5 \mathrm{MHz}$ linear phase array transducer. During the examination, patients were in the supine position, with the upper body tilted upward at about a 30 degree angle. In each vessel, peak systolic velocity (PSV), defined as the highest velocity of blood flow during the systolic phase of the cardiac cycle and the end diastolic velocity (EDV), defined as the velocity of blood flow at the end of the diastolic phase of the cardiac cycle were measured in both eyes of each glaucoma patient. Throughout the entire experimental procedure, systemic blood pressure and heart rate were recorded at 3 minute intervals by means of an automatic device (Mobil-O-Graph, IEM GmbH, Stolberg, Germany). Readings obtained during relevant variations in blood pressure were excluded.

\section{Blood flow measurement in the neuroretinal rim of the optic nerve}

Blood flow in the neuroretinal rim of the optic nerve was determined using laser Doppler flowmetry technique (LDF). ${ }^{15-17}$ The patients were seated in front of a Topcon fundus camera (Model TRC, Topcon; Tokyo, Japan). The instrument (laser Doppler flowmeter, Oculix SA, Arbaz, Switzerland) contains three light sources: a probing laser beam (wavelength: $670 \mathrm{~nm}$; power: $26 \mu \mathrm{W}$ ) for measurement (lasting 10-20 seconds, the power was well within the 1986 ANSI Z136.1 American National Standard of $107 \mu \mathrm{W}$ for a
20 minute exposure and $25 \mu \mathrm{W}$ for infinite exposure at this wavelength), a red free annulus of light to illuminate the fundus for viewing, and a fixation target. The fixation target is a $670 \mathrm{~nm}$ diode laser coupled to an optical fibre on a micromanipulator stage that allows positioning of the optic disc in the centre of the visible field.

The fundus camera was adjusted to produce an evenly illuminated focused view of the fundus. The probing laser beam was positioned with a rotatable biprism to pass through the inferior sector of the pupil and was focused to a distinct spot of $\sim 160 \mu \mathrm{m}$ in diameter on the temporal inferior neuroretinal rim, a relatively large area with good chances to allow such a measurement, distant from large calibre vessels. Patients with much damage to the temporal inferior neuroretinal rim precluding proper measurement in neural tissue were excluded. The fixation target was used to position the subject's fundus relative to the probing laser beam. An optical fibre detector with an effective $160 \mu \mathrm{m}$ diameter aperture located in the optic disc image plane of the fundus camera was positioned over the image of the spot illuminated by the probing laser beam. The detector collects the laser light scattered from the optic disc (Doppler shifted and nonshifted light) and delivers it to an external photomultiplier tube. A continuous real time digital signal processor calculates the fast Fourier transformed power spectrum. Blood flow parameters were determined by analysing the LDF signal with a NeXT (Redwood City, CA, USA) computer, using an algorithm (BPM403A, Vasamedics, Minneapolis; Periflux PF3, Perimed, Stockholm, Sweden) based on Bonner and Nossal's photon diffusion theory. ${ }^{15}{ }^{16}$ The calculated parameters (velocity, volume, and flux) are based on blood particle velocity and are related to each other through the relationship flux $=$ constant $\times$ volume $\times$ velocity. Each parameter, although given in arbitrary units, varies linearly with respect to changes in blood flow. ${ }^{17-22}$ In the present study, only the parameter flux was considered for further analysis

\section{Blood flow measurement in the choroid}

Choroidal blood flow was also determined using a method based on the laser Doppler flowmetry technique. A new device able to measure subfoveal choroidal blood flow in the fovea has been developed..$^{23}$ The optical system is based on a confocal arrangement. A polarised laser source $(\lambda=785 \mathrm{~nm}$, $100 \mathrm{~m}$ ) is relayed with a $1: 1$ optical system (laser beam at the cornea: width $=1.3 \mathrm{~mm}$, power $=90 \mu \mathrm{W})$ and focused at the subject's retina (spot in the retinal image plane $=10-20 \mu \mathrm{m}$ in diameter, optical thickness of confocal layer $=300 \mu \mathrm{m}$ ). The point laser source, the point of illumination of the fovea, and the detecting optical fibre are located in conjugated planes. The scattered light is collected by an optical system organised with six fibres arranged circularly around the central fixation point along a circle of diameter of $180 \mu \mathrm{m}$ (within the avascular zone of the fovea). The photocurrent from the photodetector is Fourier transformed and the haemodynamic parameters flux, volume, and velocity are processed as outlined above. As for optic nerve laser Doppler flowmetry, each parameter varies linearly with respect to changes in blood flow. ${ }^{24}$

The subjects fixated the red light spot within the ocular and adjusted the focus by turning the ocular. The ocular to cornea distance was set between 1.5 and $2 \mathrm{~cm}$. A constant very low level artificial room illumination was used throughout all the experiments. A stable DC during a recording was used as a criterion of proper fixation..$^{24}$ One measurement of 10-20 seconds was obtained for each eye. Again, only the parameter flux was considered for further analysis. 


\section{Statistics}

The blood pressure monitoring, the diurnal IOP curve, the CDI and laser Doppler measurements were obtained during the same 24 hour interval for each patient. Haemodynamic parameters were studied after a light breakfast and patients were asked to refrain from alcohol and caffeine for 12 hours before the measurements. A resting period of at least 30 minutes was scheduled before each haemodynamic measurement. Each time, stable baseline conditions were established, based on repeated measurements of blood pressure. Based on ocular blood flow data (one parameter per vascular bed in each eye: EDV in the ophthalmic artery and the central retinal artery as well as the LDF parameter flux of the neuroretinal rim of the optic nerve and the choroid) from both eyes of each patient, two distinct groups were computed by means of cluster analysis (Statistics for Windows, StatSoft Inc, Tulsa, OK, USA). The multivariate computation in a cluster analysis allows taking into account the influence of related variables, such as data from the fellow eye. Differences between the two clusters in age, visual field damage (index "mean defect"), peak and average IOP during the diurnal tension curve, systemic blood pressure, average ocular perfusion pressure, endothelin-1 plasma level, were evaluated by the $t$ test for independent variables. Differences in sex distribution, propensity to have normal tension glaucoma (peak IOP: $<21 \mathrm{~mm} \mathrm{Hg}$ in the diurnal tension curve) or to be vasospastic (positive nailfold capillaroscopy) were evaluated by the Fisher exact test. Differences were considered statistically significant at $\mathrm{p}$ $<0.05$.

\section{RESULTS}

Among the 56 glaucoma patients, cluster analysis grouped 26 patients ( 18 women and eight men) in a first group with higher blood flow parameters (cluster 1) and 30 patients (19 women and 11 men) in a second group with lower blood flow parameters (cluster 2). Ocular blood flow data in these two clusters are displayed in table 1. The data indicate that the two groups differed mainly in choroidal and optic nerve blood flow (fig 1). The sex distribution was statistically comparable between the two groups (Fisher's exact test: $\mathrm{p}=0.78$ ). The mean age was 66 (SD 17) years for the group with high ocular blood flow values (cluster 1) and 66 (12) years for the 30 patients (cluster 2 ) showing low ocular blood flow values $(p=0.89)$. Endothelin-1 plasma levels, visual field damage, peak and average IOP during diurnal tension curve, systemic blood pressure parameters, and ocular perfusion values did not show statistically significant differences between the two groups (table 2). Twelve patients from the first cluster and 11 patients of the second cluster had normal tension glaucoma $(p=0.59)$. In contrast, while 12 patients $(46 \%)$ from the first cluster (high ocular blood flow values) showed a vasospastic response in nailfold capillaroscopy, such a response was observed in 24 patients

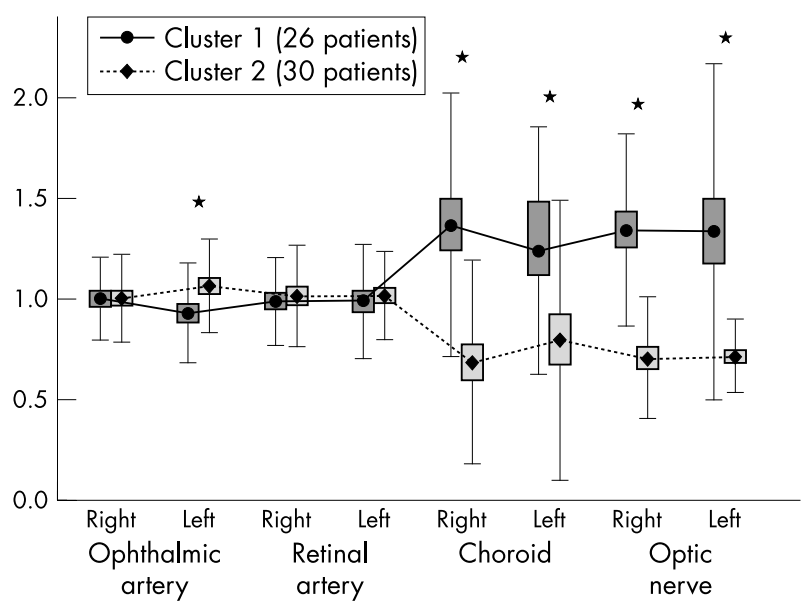

Figure 1 Based on ocular blood flow parameters two groups were computed by means of a cluster analysis. The two groups differed mainly in choroidal and optic nerve blood flow. *Indicates significantly different parameters; ophthalmic artery stays for end diastolic velocity in the ophthalmic artery of the right and left eyes respectively; retinal artery stays for end diastolic velocity in the central retinal artery of the right and left eyes respectively; choroid stays for the choroidal laser Doppler flowmetry parameter flux in the right and left eyes respectively; optic nerve stays for the optic nerve laser Doppler flowmetry parameter flux in the right and left eyes, respectively. The variables were standardised for the mean value among the 56 glaucoma patients. The boxes represent the standard error of the mean and the whiskers represent the standard deviation.

$(80 \%)$ from the cluster with low ocular blood flow values $(\mathrm{p}=0.0121)$.

\section{DISCUSSION}

Two clusters of patients with open angle glaucoma were computed based on ocular blood flow parameters. Although some uncertainty regarding the nature of the parameters measured with the devices used in the present study persists, ${ }^{25}$ all are related to ocular blood flow. In the present study, the two computed groups differed mainly in choroidal and optic nerve blood flow parameters. Between these two clusters, no differences in sex distribution, propensity to have normal tension glaucoma, age, endothelin-1 plasma levels, visual field damage, intraocular pressure, systemic blood pressure parameters, or ocular perfusion values were observed. The only statistically significant difference between the two groups, besides the parameters used to compute the two clusters, was the propensity to show a vasospastic response in nailfold capillaroscopy, with $46 \%$ of the patients of the group with high values of ocular blood flow parameters showing a capillary blood flow stop, while this proportion reached $80 \%$ among the patients with low values of ocular blood flow parameters. The present study did not address the effect of low values of blood flow parameters on the

Table 1 Ocular haemodynamic parameters (mean (SD))

\begin{tabular}{|c|c|c|c|}
\hline & Cluster 1 (26 patients) & Cluster 2 (30 patients) & p Value \\
\hline $\begin{array}{l}\text { EDV }(\mathrm{cm} / \mathrm{s}) \text { in the right ophthalmic artery } \\
\text { EDV }(\mathrm{cm} / \mathrm{s}) \text { in the left ophthalmic artery } \\
\text { EDV }(\mathrm{cm} / \mathrm{s}) \text { in the right central retinal artery } \\
\text { EDV }(\mathrm{cm} / \mathrm{s}) \text { in the left central retinal artery } \\
\text { Choroidal flux (AU) in the right eye } \\
\text { Choroidal flux (AU) in the left eye } \\
\text { Optic nerve flux (AU) in the right eye } \\
\text { Optic nerve flux (AU) in the left eye }\end{array}$ & $\begin{array}{l}7.78(1.59) \\
7.15(1.90) \\
2.16(0.48) \\
2.21(0.64) \\
20.32(9.76) \\
19.12(9.50) \\
38.29(13.62) \\
41.23(25.89)\end{array}$ & $\begin{array}{l}7.79(1.71) \\
8.18(1.78) \\
2.21(0.55) \\
2.28(0.49) \\
10.18(7.56) \\
12.26(10.72) \\
20.17(8.60) \\
22.07(5.66)\end{array}$ & $\begin{array}{l}0.97 \\
0.041 \\
0.69 \\
0.65 \\
<0.0001 \\
0.015 \\
<0.0001 \\
0.00023\end{array}$ \\
\hline
\end{tabular}


Table 2 Endothelin-1 plasma levels, visual field damage, intraocular pressure, and systemic blood pressure (mean (SD))

\begin{tabular}{|c|c|c|c|}
\hline & Cluster 1 (26 patients) & Cluster 2 (30 patients) & p Value \\
\hline Endothelin-1 plasma level (pg/ml) & $2.90(0.76)$ & $2.90(0.81)$ & 0.98 \\
\hline$M D$ in right eyes $(\mathrm{dB})$ & $7.65(6.87)$ & $7.95(6.63)$ & 0.87 \\
\hline$M D$ in left eyes $(\mathrm{dB})$ & $8.32(7.29)$ & $10.36(18.65)$ & 0.60 \\
\hline Peak IOP in right eyes $(\mathrm{mm} \mathrm{Hg})$ & $21.88(6.50)$ & $21.50(5.12)$ & 0.81 \\
\hline Peak IOP in left eyes $(\mathrm{mm} \mathrm{Hg})$ & $22.19(7.50)$ & $21.37(4.35)$ & 0.61 \\
\hline Average IOP in right eyes $(\mathrm{mm} \mathrm{Hg})$ & $14.63(2.00)$ & $14.37(2.78)$ & 0.70 \\
\hline Average IOP in right eyes $(\mathrm{mm} \mathrm{Hg})$ & $14.61(3.47)$ & $14.87(2.85)$ & 0.76 \\
\hline Average systolic 24 hours blood pressure $(\mathrm{mm} \mathrm{Hg})$ & $118.71(19.04)$ & $118.72(18.23)$ & 0.99 \\
\hline Average diastolic 24 hours blood pressure $(\mathrm{mm} \mathrm{Hg})$ & $74.04(9.68)$ & $74.04(9.81)$ & 0.99 \\
\hline Average systolic day-time blood pressure $(\mathrm{mm} \mathrm{Hg})$ & $128.66(20.29)$ & $127.95(17.41)$ & 0.89 \\
\hline Average diastolic day-time blood pressure $(\mathrm{mm} \mathrm{Hg})$ & $81.69(9.98)$ & $81.18(9.27)$ & 0.85 \\
\hline Average systolic night-time blood pressure $(\mathrm{mm} \mathrm{Hg})$ & $111.59(18.50)$ & $112.60(20.38)$ & 0.85 \\
\hline Average diastolic night-time blood pressure $(\mathrm{mm} \mathrm{Hg})$ & $68.51(9.49)$ & $69.28(11.41)$ & 0.79 \\
\hline Nocturnal drop $(\%)$ of systolic blood pressure & $13.15(5.90)$ & $12.04(8.94)$ & 0.59 \\
\hline Nocturnal drop (\%) of diastolic blood pressure & $15.99(7.71)$ & $14.60(10.33)$ & 0.58 \\
\hline Ocular perfusion pressure in right eyes & $50.27(7.35)$ & $50.14(7.47)$ & 0.95 \\
\hline Ocular perfusion pressure in left eyes & $50.29(7.84)$ & $49.64(7.25)$ & 0.75 \\
\hline
\end{tabular}

$M D=$ visual field index "mean defect"; $I O P$ = intraocular pressure values refer to values obtained during the diurnal tension curve; blood pressure values refer to values obtained during 24 hour blood pressure monitoring.

prognosis of glaucomatous disease, but suggested a statistical association between altered ocular blood flow parameters and a vasospastic propensity. This observation is of importance, indicating that systemic blood flow alterations are related to ocular blood flow disturbances in glaucoma.

We used a somewhat unusual approach in the present analysis. In contrast with traditional hypothesis testing designed to verify differences between groups of patients, an exploratory data analysis was employed. Parameters of ocular blood flow were used to compute two groups, using cluster analysis, a multivariate exploratory technique designed specifically to identify patterns in multivariate data sets. Theoretically, any set of variables such as intraocular pressure or visual field damage can be used to compute clusters, each approach addressing a different question. Computationally, cluster analysis may be thought of as analysis of variance "in reverse." The computation will start with random clusters, and then move patients between those clusters with the goal to (1) minimise variability within clusters and (2) maximise variability between clusters. In contrast with traditional hypothesis testing where groups of patients are defined based on obvious criteria, a cluster analysis allows us to define groups of patients based on more complex characteristics. Once the groups are defined, they can be compared with regard to parameters other than those used to compute the clusters.

The present cluster analysis yielded intriguing observations. The most marked differences were noted for choroidal and optic nerve blood flow parameters (both eyes showing the same trend). End diastolic velocity in the ophthalmic artery (probably the least "ocular" of all tested vessels) was higher, but only borderline significantly and only in the left eyes of patients with generally low ocular blood flow. Therefore, this finding does, in our opinion, not invalidate our consideration of the two clusters as one group with low ocular blood flow and one group with high ocular blood flow. Patients with low values of ocular blood flow parameters did not have more advanced visual field damage, suggesting that blood flow alterations do not, at least not only, occur secondary to visual field damage. The same patients were not necessarily patients with normal tension glaucoma and did not have markedly lower untreated IOP readings in a diurnal tension curve, suggesting that ocular blood flow alterations are not, at least not only, secondary to increased IOP. Furthermore, low values of ocular blood flow parameters were not related to low systemic blood pressure, suggesting that ocular blood flow alterations occur not only in response to systemic perturbations. The only positive association with low values of ocular blood flow parameters was observed with a vasospastic responsiveness of the nailfold capillaries. The fact that up to half of the patients with high values of ocular blood flow parameters show a vasospastic responsiveness of the nailfold capillaries suggests that the presence of systemic vasospastic responsiveness does not necessarily mean that ocular blood flow is altered. This is also in accordance with the relative high frequency of a vasospastic propensity in a healthy population. ${ }^{26}$ Nevertheless, the fact that four out of five patients with low values of ocular blood flow parameters showing a vasospastic responsiveness of the nailfold capillaries, suggests an association between altered ocular blood flow and vasospastic propensity. It is unlikely that the latter association is because the observed lower values of ocular blood flow parameters are brought about by a systemic vasoconstrictive response to an external perturbation. However, although this interpretation is purely speculative, it is possible that similar phenomena occur within the ocular vascular bed of some, but not all, glaucoma patients with a vasospastic responsiveness of the nailfold capillaries, leading ultimately to lower blood flow parameters. Most importantly, results of a cluster analysis are to be understood as giving a hunch of what should be investigated. An inferring interpretation of the results would not be appropriate and the present findings need further confirmation.

Regarding risk factors in glaucoma, the differentiation between mechanical damage, as a consequence of IOP, ${ }^{27}$ and vascular theories, considering glaucomatous optic neuropathy as a consequence of insufficient blood supply, ${ }^{28}$ had little therapeutic impact so far, as IOP reduction was the only intervention available for treatment of glaucoma. Clinical intervention studies have proved the role of IOP and the benefits of IOP lowering treatment. ${ }^{29}{ }^{30}$ Given the success of IOP reduction, consideration of ancillary risk factors may seem distracting. However, once pharmacological means and laser surgery have failed, what remains is a filtering procedure with all its risks and possible complications. Elucidating how other factors influence the course of glaucomatous disease may open new therapeutic avenues as welcome alternatives to surgical treatment.

\section{Authors' affiliations}

M Emre, S Orgül, K Gugleta, J Flammer, University Eye Clinic, Basel, Switzerland

Financial support: Swiss National Foundation (32-059094.99). 


\section{REFERENCES}

1 Fechtner R, Weinreb RN. Mechanisms of optic nerve damage in primary open-angle glaucoma. Surv Ophthalmol 1994;39:23-42.

2 Flammer J, Orgul S. Optic nerve blood-flow abnormalities in glaucoma. Prog Ret Eye Res 1998;17:267-89.

3 Orgul S, Flammer J, Gasser P. Female preponderance in normal-tension glaucoma. Ann Ophthalmol 1995;27:355-9.

4 Moriya S, Sugiyama T, Shimizu K, et al. Low-tension glaucoma and endothelin (ET-1). Folia Ophthalmol Jpn 1992;43:554-9.

5 Kaiser HJ, Flammer J, Wenk M, et al. Endothelin-1 plasma levels in normaltension glaucoma: abnormal response to postural changes. Graefes Arch Clin Exp Ophthalmol 1995;233:484-8.

6 Kaiser HJ, Flammer J. Systemic hypotension: a risk factor for glaucomatous damage? Ophthalmologica 1991;203:105-8

7 Kaiser HJ, Flammer J, Graf T, et al. Systemic blood pressure in glaucoma patients. Graefes Arch Clin Exp Ophthalmol 1993;231:677-80.

8 Hayreh SS, Zimmerman MB, Podhajsky P, et al. Nocturnal arterial hypotension and its role in optic nerve head and ocular ischemic disorders. Am J Ophthalmol 1994;117:603-24.

9 Tielsch JM, Katz J, Sommer A, et al. Hypertension, perfusion pressure, and primary open-angle glaucoma. A population-based assessment. Arch Ophthalmol 1995; 1 13:216-21.

10 Graham SL, Drance SM, Wiisman K, et al. Ambulatory blood pressure monitoring in glaucoma. The nocturnal dip. Ophthalmology 1995;102:61-9.

11 Gasser P, Flammer J. Blood-cell velocity in the nailfold capillaries of patients with normal-tension and high-tension glaucoma. Am J Ophthalmol $1991 ; 111: 585-8$

12 Barton M, Shaw S, d'Uscio LV, et al. Angiotensin II increases vascular and renal endothelin- 1 and functional endothelin converting enzyme activity in vivo: role of ETA receptors for endothelin regulation. Biochem Biophys Res Commun 1997;238:861-5.

13 Flammer J, Jenni A, Bebie $\mathrm{H}$, et al. The Octopus glaucoma G1 program. Glaucoma 1987;9:67-72.

14 Gherghel D, Orgul S, Gugleta K, et al. Relationship between ocular perfusion pressure and retrobulbar blood flow in patients with glaucoma with progressive damage. Am J Ophthalmol 2000;130:597-605.
15 Bonner R, Nossal R. Model for laser Doppler measurements of blood flow in tissue. Appl Opt 1981;20:2097-107.

16 Bonner RF, Nossal R. In: Shepherd AP, Oberg P, eds. Laser-Doppler blood flowmetry. Boston: Kluwer Academic Publishers, 1990:17-46.

17 Riva CE, Harino S, Petrig BL, et al. Laser Doppler flowmetry in the optic nerve. Exp Eye Res 1992;55:499-506.

18 Smits GJ, Roman RJ, Lombard JH. Evaluation of laser-Doppler flowmetry as a measure of tissue blood flow. J Appl Physiol 1986;61:666-72.

19 Fukuda O, Endo S, Kuwayama N, et al. The characteristics of laser-Doppler flowmetry for the measurement of regional cerebral blood flow. Neurosurgery 1995;36:358-64.

20 Rundquist I, Smith QR, Michel ME, et al. Sciatic nerve blood flow measured by laser Doppler flowmetry and [14C]iodoantipyrine. Am J Physiol 1985;248(3 Pt 2):H311-17.

21 Schabauer AM, Rooke TW. Cutaneous laser Doppler flowmetry: applications and findings. Mayo Clin Proc 1994;69:564-74.

22 Lausten GS, Kiaer T, Dahl B. Laser Doppler flowmetry for estimation of bone blood flow: studies of reproducibility and correlation with microsphere technique. J Orthop Res 1993;1 1:573-80.

23 Geiser MH, Diermann U, Riva CE. Compact laser Doppler choroidal flowmeter. J Biomed Opt 1999;4:459-64.

24 Gugleta K, Orgul S, Flammer I, et al. Reliability of confocal choroidal laser Doppler flowmetry. Invest Ophthalmol Vis Sci 2002:723-8.

25 Hayreh SS. Evaluation of optic nerve head circulation: review of the methods used. J Glaucoma 1997;6:319-30.

26 Hasler PW, Orgul S, Gugleta K, et al. Vascular dysregulation in the choroid of subjects with acral vasospasm. Arch Ophthalmol 2002;120:302-7.

27 Yan DB, Coloma FM, Metheetrairut A, et al. Deformation of the lamina cribrosa by elevated intraocular pressure. Br J Ophthalmol 1994;78:643-8.

28 Flammer J. The vascular concept of glaucoma. Surv Ophthalmol 1994:38(suppl):3-6.

29 Collaborative Normal-Tension Glaucoma Study Group. The effectiveness of intraocular pressure reduction in the treatment of normal tension glaucoma. Am J Ophthalmol 1998;126:498-505.

30 The AGIS Investigators. The advanced glaucoma intervention study (AGIS): 7. The relationship between control of intraocular pressure and visual field deterioration. Am J Ophthalmol 2000;130:429-40. 